\title{
DISEÑO BASADO EN DESPLAZAMIENTOS DE ESTRUCTURAS ESENCIALES
}

\author{
Gerardo Díaz Martínez $^{(1)}$, Amador Terán Gilmore ${ }^{(2)}$ y Carlos Reyes Salinas $^{(3)}$
}

\begin{abstract}
RESUMEN
Mediante el uso de sistemas equivalentes de un grado de libertad, se evalúa la pertinencia del uso que actualmente se da al factor de importancia durante el diseño sísmico de estructuras esenciales desplantadas en terreno blando. Dada la alta vulnerabilidad que a partir de sus demandas de distorsión muestran algunos de estos sistemas, se propone una metodología de diseño basada en desplazamientos para la concepción de sistemas estructurales que sean capaces de controlar adecuadamente los niveles de daño estructural y no estructural en instalaciones esenciales. La metodología es aplicable a edificios estructurados con base en marcos rígidos (también conocidos como continuos) y contempla la acción de eventos sísmicos intensos. Para mantener los niveles de daño estructural y no estructural dentro de niveles admisibles, se plantea el control simultáneo de las demandas máximas de rotación plástica y de distorsión de entrepiso. Los ejemplos que se presentan muestran que el uso de la metodología resulta en estructuras esenciales capaces de satisfacer el nivel de desempeño de Ocupación Inmediata ante la ocurrencia del sismo de diseño.
\end{abstract}

Palabras clave: factor de importancia; estructuras esenciales; diseño por desempeño; ocupación inmediata

\begin{abstract}
The pertinence of using an importance factor during the seismic design of essential facilities located in soft soil is assessed through the use of equivalent single-degree-of-freedom systems. Because of the large vulnerability shown by some of these systems in terms of their drift demands, a displacement-based methodology aimed at the conception of structural systems capable of adequately controlling the level of structural and non-structural damage in essential facilities is formulated. The methodology is applicable to buildings structured with moment-resistant frames; and contemplates the occurrence of severe ground motions. To control the levels of structural and non-structural damage within acceptable thresholds, the simultaneous control of the maximum plastic rotation and inter-story drift index demands is formulated. The examples developed in the paper show that the use of the methodology results in essential facilities that are capable of satisfying the Immediate Occupancy performance level after the occurrence of the design ground motion.
\end{abstract}

Keywords: importance factor; essential facilities; performance-based design; immediate occupancy

Artículo recibido el 17 de mayo de 2012 y aprobado para su publicación el 08 de abril de 2013. Se aceptarán comentarios y/o discusiones hasta cinco meses después de su publicación.

(1) Estudiante de Posgrado, Universidad Autónoma Metropolitana. Av. San Pablo 180, Col. Reynosa Tamaulipas, México 02200, D.F. email: gdm@ correo.azc.uam.mx

(2) Profesor, Departamento de Materiales. Universidad Autónoma Metropolitana. Av. San Pablo 180, Col. Reynosa Tamaulipas, México 02200, D.F. tga@ correo.azc.uam.mx

(3) In Memoriam 


\section{INTRODUCCIÓN}

El desempeño sísmico insatisfactorio de algunas estructuras diseñadas conforme a los reglamentos de diseño sísmico actuales, ha preocupado al medio de la ingeniería estructural. Esto ha cobrado particular importancia a partir de las grandes pérdidas materiales y económicas que han resultado de eventos sísmicos severos, como los de México 1985, Northridge 1994, Kobe 1995, Taiwán 1999, Sichuan 2008 y Chile 2010. Dado el nivel inesperadamente alto de pérdidas, se han formulado enfoques integrales de diseño sísmico, tal como el de diseño por desempeño, que enfatizan la necesidad de un control explícito de la respuesta dinámica de las estructuras.

El origen del mal desempeño sísmico de las estructuras puede encontrarse en algunas de las deficiencias y lagunas existentes en los procedimientos actuales de diseño sísmico. En particular, el énfasis que se pone en la resistencia de la estructura, y la falta de atención a demandas que pueden ser relevantes para su desempeño sísmico hacen imposible para el diseñador considerar todos los aspectos de importancia durante el diseño. Actualmente, la función del ingeniero estructural ya trasciende el diseño de estructuras que no fallen, y alcanza la obligación de satisfacer las muchas necesidades y expectativas socioeconómicas que han surgido alrededor de la construcción de obras de ingeniería civil.

Se ha observado que los niveles de daño estructural y no estructural que un edificio exhibe después de una excitación sísmica, dependen de los valores que durante la misma adquiere el desplazamiento máximo (Qi y Moehle, 1991; Bertero y Bertero, 1992; Priestley et al., 2007). De igual manera, se ha llegado a la conclusión de que algunos contenidos de las estructuras son susceptibles a las demandas de velocidad y aceleración (Villaverde, 1997; Takahashi y Shiohara, 2004). En particular, mientras estos parámetros de respuesta (desplazamiento, velocidad y aceleración) se incrementan, mayor es el nivel de daño o degradación esperado en la estructura y sus contenidos. Dentro de este contexto, las propiedades estructurales que deben suministrarse a una estructura deben ser tales que controlen su respuesta lateral dentro de umbrales que sean congruentes con el nivel de daño o desempeño deseado para los elementos estructurales, elementos no estructurales y el contenido de la estructura. La tabla 1 resume valores de distorsión de entrepiso (DI) que pueden ser asociados a los niveles de desempeño de Operación y Prevención de Colapso de marcos rígidos de acero y concreto reforzado (Reyes, 2000).

Tabla 1. Distorsión de entrepiso que causa daño en marcos rígidos de acero y concreto reforzado

\begin{tabular}{ccc}
\hline \multirow{2}{*}{ Sistema Estructural } & \multicolumn{2}{c}{ Distorsión } \\
\cline { 2 - 3 } & Operación & Colapso Incipiente \\
\hline Marcos de concreto con detallado sísmico & 0.005 & 0.04 \\
Marcos de concreto sin detallado sísmico & 0.0073 & 0.02 \\
Marcos de acero con detallado sísmico & 0.0055 & 0.03 \\
Marcos de acero sin detallado sísmico & 0.0074 & 0.02 \\
\hline
\end{tabular}

A pesar de las consideraciones especiales hechas actualmente para el diseño sísmico de las estructuras esenciales, éstas presentan alta vulnerabilidad ante la acción de movimientos de terreno de gran intensidad (Terán-Gilmore y Jirsa, 2010). En términos generales, para que una instalación esencial cumpla con el nivel de desempeño de Ocupación Inmediata debe contar con elementos estructurales que provean resistencia y rigidez suficiente, y elementos no estructurales que permanezcan libres de daño. Para cuantificar la resistencia y rigidez lateral de diseño, los requerimientos normativos actuales proponen el uso de un factor de importancia para incrementar las fuerzas laterales de diseño. En el caso particular del Reglamento de Construcciones del Distrito Federal (RCDF, 2004), el factor de importancia toma un valor de 1.5 . 
Estudios previos sugieren que el uso de un factor de importancia de 1.5 para el diseño de estructuras esenciales localizadas en terreno firme conduce a sistemas capaces de satisfacer el nivel de desempeño de Ocupación Inmediata siempre y cuando su resistencia lateral se estime a partir de un espectro de diseño asociado a una ductilidad máxima de 2 (Terán et al., 2010). Dentro de un contexto similar, el trabajo que aquí se presenta evalúa el efecto de utilizar un factor de importancia de 1.5 durante el diseño sísmico de estructuras esenciales ubicadas en la Zona del Lago del Distrito Federal. Para ello, se estiman mediante el uso de sistemas equivalentes de un grado de libertad las demandas sísmicas en estructuras esenciales con una amplia gama de propiedades estructurales. Los niveles de daño estructural y no estructural se evalúan a partir de las demandas máximas de rotación plástica y distorsión de entrepiso, respectivamente. Debido al desempeño sísmico deficiente observado para varios de los sistemas estudiados, se plantea una metodología de diseño basada en desplazamientos para la concepción y diseño preliminar de instalaciones esenciales estructuradas con base en marcos rígidos. El uso de la metodología se ejemplifica mediante el diseño de dos versiones del sistema estructural de un edificio de ocho pisos. A través de la evaluación del desempeño sísmico de las estructuras diseñadas, se concluye que la metodología representa una herramienta útil para la concepción y diseño preliminar de estructuras esenciales ubicadas en terreno blando.

En términos de alcance, el trabajo se enfoca a presentar y evaluar el uso de la metodología de diseño para el caso de estructuras esenciales ubicadas en la Zona del Lago del Distrito Federal. La metodología aplica al caso de edificios estructurados con base en marcos rígidos regulares de acero, y no considera explícitamente el desempeño sísmico de los contenidos vulnerables a altas demandas de velocidad y aceleración.

\section{EVALUACIÓN DEL DESEMPEÑO SÍSMICO DE ESTRUCTURAS ESENCIALES DESPLANTADAS EN LA ZONA DEL LAGO DEL DISTRITO FEDERAL}

La siguiente sección describe un sistema de un grado de libertad que se utilizará más adelante para el desarrollo de un estudio paramétrico de la respuesta de estructuras esenciales ubicadas en la Zona del Lago del Distrito Federal. Para ello, se considera que es posible utilizar un modelo equivalente de un grado de libertad para estimar las demandas de deformación, tanto a nivel global como a nivel local, de edificios esenciales estructurados con base en marcos rígidos que exhiben regularidad de geometría, masa, rigidez y resistencia, tanto en planta como en elevación.

\section{Modelo equivalente de un grado de libertad}

En algunos casos es posible estimar la respuesta de un edificio regular a partir de sistemas simples. En el caso particular de este trabajo, se utiliza un sistema equivalente de un grado de libertad que se plantea conforme a lo siguiente (Terán, 2004, Terán et al., 2010):

a) Se definen las propiedades relevantes del edificio, tal como su altura total $(H)$, periodo fundamental de vibración $(T)$, coeficiente sísmico ( $c$, definido como el cortante basal último que desarrolla el sistema normalizado por su peso), y porcentaje de amortiguamiento crítico $(\xi)$. La figura 1 ilustra la definición del coeficiente sísmico, $W$ denota el peso total del edificio.

b) Se establece el sistema equivalente de un grado de libertad asignando a su periodo, coeficiente sísmico y porcentaje de amortiguamiento crítico, los valores de $T, c$ y $\xi$, respectivamente, establecidos para el edificio. 


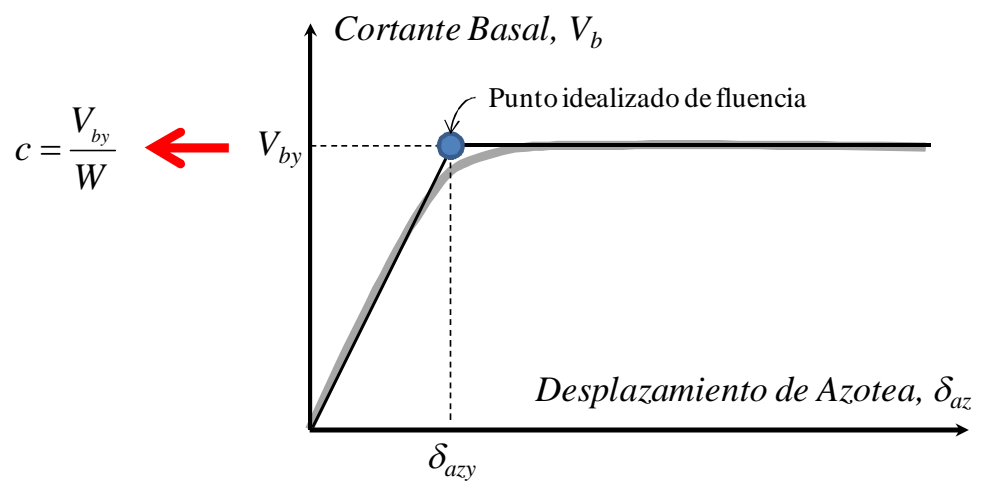

Figura 1. Definición de coeficiente sísmico a partir de curva de capacidad

Una vez establecido el sistema equivalente de un grado de libertad, se le sujeta a la acción del sismo bajo consideración, y se establecen sus demandas máximas de ductilidad $\left(\mu_{S l G L}\right)$ y de desplazamiento lateral $\left(\delta_{S I G L}\right)$. En función del número de pisos del edificio $(N)$ y el valor de $\mu_{S I G L}$, es posible establecer su demanda máxima de desplazamiento de azotea conforme a lo siguiente:

$$
\delta_{\max }=\alpha \delta_{S 1 G L}
$$

donde $\alpha$ es un factor que se calibra a partir del estudio analítico de edificios con sistemas estructurales similares al que se estudia. Con base en las recomendaciones del FEMA 306 (Applied Technology Council, 1998) y en los estudios llevados a cabo por Terán (Terán, 2004), la tabla 2 presenta valores de $\alpha$ que pueden utilizarse para el caso de edificios estructurados con base en marcos rígidos regulares. En caso de que el valor de $\mu_{S I G L}$ se encuentre en el rango que va de 1 a 2 , es necesario interpolar con base en los valores incluidos en la tabla.

Tabla 2. Valores de $\alpha$ para marcos rígidos regulares

\begin{tabular}{ccc}
\hline Número & \multicolumn{2}{c}{$\alpha$} \\
\cline { 2 - 3 } de pisos & $\mu_{S I G L}=1$ & $\mu_{S I G L}=2+$ \\
\hline 1 & 1.00 & 1.00 \\
2 & 1.20 & 1.10 \\
3 & 1.30 & 1.20 \\
4 & 1.35 & 1.20 \\
$5+$ & 1.40 & 1.20 \\
\hline
\end{tabular}

Una vez que se tiene la máxima demanda de desplazamiento de azotea, es posible hacer una estimación de la máxima demanda de distorsión de entrepiso $\left(D I_{\max }\right)$ :

$D I_{\max }=\frac{\delta_{\max } C O D}{H}$

donde $C O D$ es un coeficiente que considera que la demanda de distorsión de entrepiso no es constante a lo largo de la altura del edificio. De manera similar a lo discutido para el parámetro $\alpha, C O D$ debe calibrarse a partir del estudio analítico de sistemas estructurales similares al que se estudia. Con base en las discusiones planteadas por diversos autores (Qi y Moehle, 1991 y Bertero et al., 1991), y en los estudios presentados por otros (Terán, 2004), la tabla 3 ofrece valores de COD para marcos rígidos regulares. Mientras que en el caso de que el valor de $\mu_{S I G L}$ sea menor que 1 debe usarse un $C O D$ igual a 1.2, cuando 
esta demanda se encuentre en el rango que va de 1 a 2 , es necesario interpolar con base en los valores incluidos en la tabla.

Tabla 3. Valores de $C O D$ para marcos rígidos regulares

\begin{tabular}{cc}
\hline$\mu_{S I G L}$ & $C O D$ \\
\hline 1 & 1.2 \\
$2+$ & $1.5 \mathrm{a} 1.8$ \\
\hline
\end{tabular}

Debido a que el nivel de daño estructural exhibe una alta correlación con las demandas de deformación no lineal, en el presente estudio se considera que un sistema que ha permanecido elástico $\left(\mu_{S I G L} \leq 1\right)$ durante la excitación sísmica no sufre daño estructural. Debido a lo anterior, es necesario delimitar las porciones de deformación elástica y plástica involucradas en el valor de $D I_{\max }$ que corresponde a un sistema estructural que ha incursionado en su rango plástico de comportamiento $\left(\mu_{S I G L}>\right.$ 1). Para ello, es necesario establecer primero la máxima distorsión elástica en el edificio (la cual corresponde al desplazamiento $\delta_{a z y}$ mostrado en la figura 1):

$$
D I_{\max }^{e l}=\frac{\delta_{a z y} C O D}{H}
$$

La componente plástica de la distorsión máxima de entrepiso puede entonces estimarse como:

$D I_{\text {max }}^{p l}=D I_{\text {max }}-D I_{\text {max }}^{e l}$

Para ilustrar la pertinencia de estimar las demandas de distorsión de entrepiso en marcos rígidos regulares a partir del uso del modelo de un grado de libertad discutido aquí, se aplica el modelo para predecir las demandas de desplazamiento de azotea y distorsión de siete edificios cuyo sistema estructural exhibe la configuración estructural mostrada en la figura 2. Conforme a lo discutido en detalle por Terán (1998), los edificios fueron diseñados conforme a la versión anterior del Reglamento de Construcciones para el Distrito Federal y sus Normas Técnicas Complementarias, para un coeficiente de comportamiento sísmico de 4 y la Zona del Lago del Distrito Federal. Conforme a las consideraciones de modelado discutidas por Terán (Terán, 1998), se prepararon modelos de análisis no lineales para los edificios, y se obtuvieron sus curvas de capacidad a partir de una serie de análisis estáticos no lineales. La tabla 4 resume las principales características y propiedades estructurales establecidas para los edificios a partir de sus curvas de capacidad y su modelado no lineal.
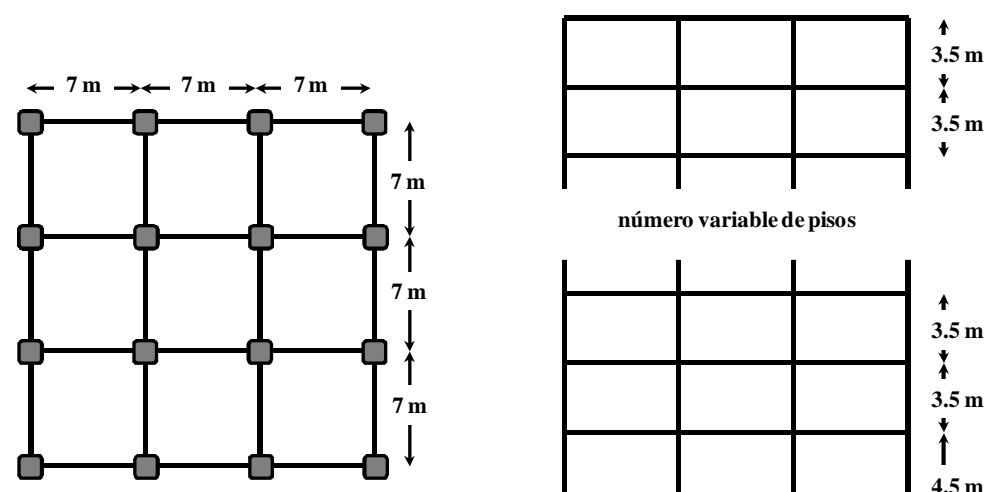

número variable de pisos

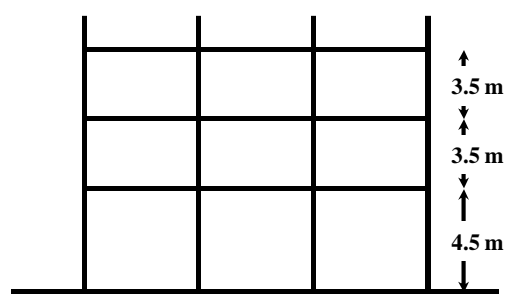

Figura 2. Configuración estructural de marcos rígidos en consideración 
Los modelos de análisis no lineal de los siete edificios fueron sujetos a la acción de la componente Este-Oeste del movimiento registrado durante 1985 en la Secretaría de Comunicaciones y Transportes (SCT EO). Vale la pena destacar que las envolventes de demandas de desplazamiento de azotea, de distorsión de entrepiso y de rotación plástica mostradas en la figura 3, fueron obtenidas bajo la consideración de que el comportamiento histerético de vigas y columnas se modela con un comportamiento elasto-plástico y que a los dos primeros modos de vibrar de cada edificio les corresponde el 5\% del amortiguamiento crítico. En cuanto a la figura 3c, ésta muestra la media de las rotaciones plásticas máximas desarrolladas en los extremos de todas las vigas ubicadas en el piso con mayores demandas plásticas $\left(\theta_{p}^{\text {prom }}\right)$.

Tabla 4. Características y propiedades estructurales de edificios bajo consideración

\begin{tabular}{ccccc}
\hline Edificio & $N$ & $H(\mathrm{~m})$ & $T(\mathrm{seg})$ & $C$ \\
\hline 1 & 4 & 13.50 & 0.83 & 0.297 \\
2 & 6 & 19.50 & 1.17 & 0.206 \\
3 & 8 & 25.50 & 1.28 & 0.211 \\
4 & 8 & 25.50 & 1.37 & 0.185 \\
5 & 10 & 31.50 & 1.46 & 0.198 \\
6 & 12 & 37.50 & 1.48 & 0.206 \\
7 & 16 & 49.50 & 1.86 & 0.196 \\
\hline
\end{tabular}
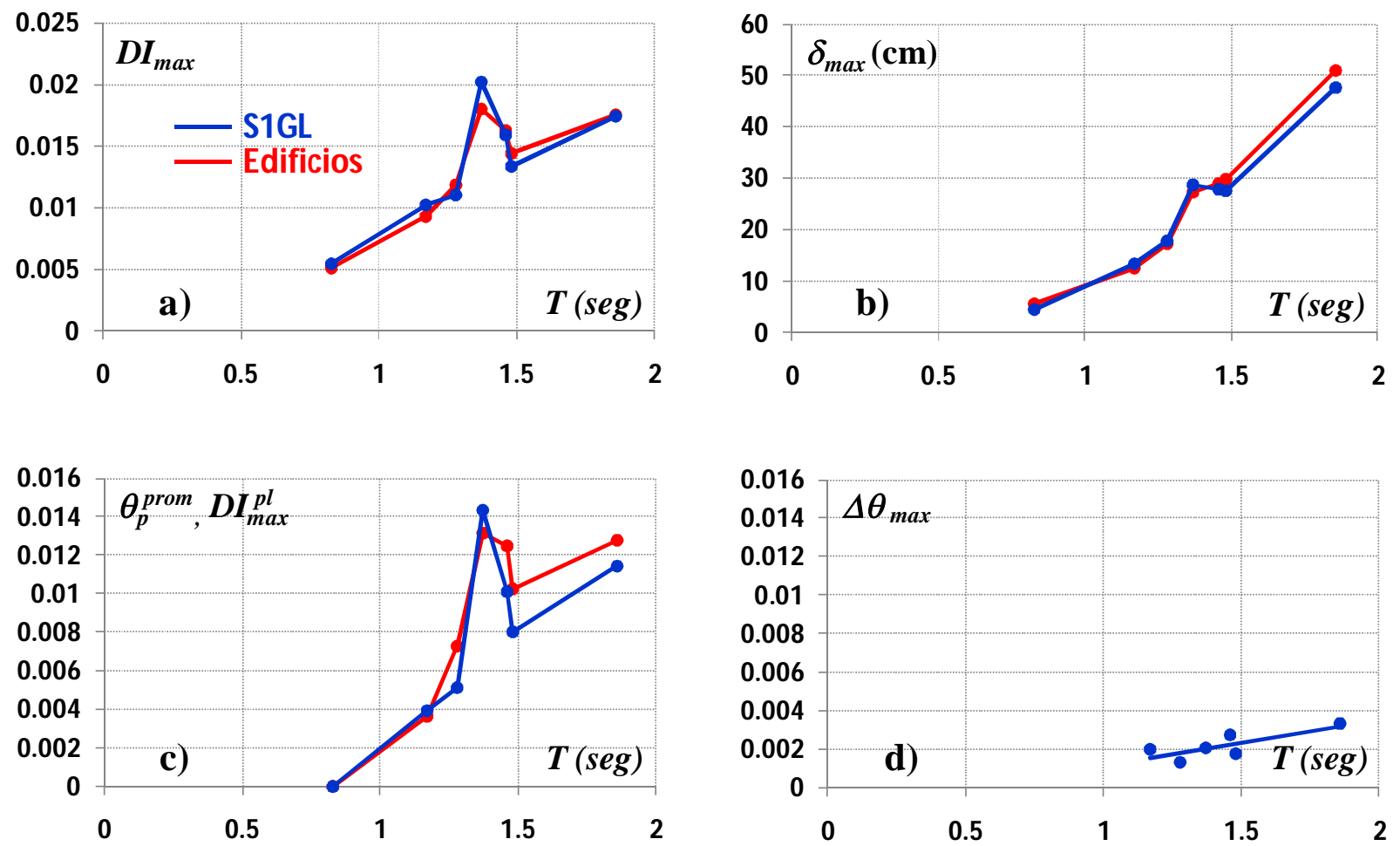

Figura 3. Demandas sísmicas: a) Distorsión máxima; b) Desplazamiento de azotea; c) Promedio de rotación plástica; d) Diferencia entre la mayor rotación plástica y la máxima distorsión plástica

Se establecieron conforme a lo discutido en este estudio, modelos equivalentes de un grado de libertad para los edificios. Por congruencia, los modelos equivalentes consideraron 5\% de 
amortiguamiento crítico y comportamiento elasto-plástico perfecto, y se sujetaron a la acción del movimiento SCT EO. Dentro de los posibles valores de COD para sistemas que exhiben comportamiento plástico $\left(\mu_{S 1 G L} \geq 2\right)$, se utilizó un valor de 1.7 (ver tabla 3 ). Note en la figura 3 que los modelos de un grado de libertad estiman de manera razonable las demandas de deformación en los edificios, tanto a nivel local como a nivel global. En cuanto a la figura 3c, la comparación presentada en ella muestra que las medias de las rotaciones plásticas máximas pueden ser estimadas de manera razonable a partir de los valores de la componente plástica de la distorsión máxima de entrepiso (ecuación 4).

Note que si la figura $3 \mathrm{c}$ muestra las medias de rotación plástica máxima para un piso, esto implica que en algunos extremos de algunas vigas la rotación plástica máxima excederá ese valor promedio. No deja de ser de interés plantear para los edificios bajo consideración una manera de establecer la máxima rotación plástica de entrepiso a partir de la componente plástica de distorsión de entrepiso ofrecida por el sistema equivalente de un grado de libertad. Para ello, se define $\Delta \theta_{\max }$ conforme a lo siguiente:

$$
\Delta \theta_{\max }=\theta_{p}^{\max }-D I_{\max }^{p l}
$$

donde $\theta_{p}^{\max }$ es la mayor entre todas las rotaciones plásticas máximas desarrolladas en las vigas del entrepiso con las mayores demandas plásticas. La figura $3 \mathrm{~d}$ muestra valores de $\Delta \theta_{\max }$ para seis de los siete edificios (el edificio de cuatro niveles permanece en su rango elástico de comportamiento cuando se le sujeta a SCT EO). Los valores de $\Delta \theta_{\max }$ tienden a caer en un rango de valores que va de 0.002 a 0.003 .

Es importante mencionar que el uso de un modelo equivalente de un grado de libertad para establecer las demandas sísmicas de sistemas estructurales de varios pisos, implica una serie de suposiciones que delimitan el alcance de los estudios aquí planteados. En particular, es importante mencionar que los valores de $\alpha$ y $C O D$ dependen de la distribución en altura de rigidez y resistencia lateral del sistema estructural. Valores específicos para estos parámetros, como los que se resumen en las tablas 2 y 3 , solo son aplicables a determinado tipo de sistemas estructurales. Dentro de este contexto, los valores propuestos ofrecen estimaciones razonables para las demandas de distorsión de entrepiso de sistemas estructurados con base en marcos rígidos regulares (como el mostrado esquemáticamente en la figura 2). En este artículo, se considera que un edificio es regular si su sistema estructural cumple con las condiciones de regularidad contempladas por la Sección 6.1 de las Normas Técnicas Complementarias para Diseño por Sismo del Reglamento de Construcciones para el Distrito Federal (RCDF, 2004). Lo anterior resulta en que los edificios bajo consideración no exhiban una respuesta torsional de importancia ni concentren de manera indebida sus demandas plásticas de deformación en unos cuantos entrepisos.

\section{Registros utilizados}

En cuanto a los movimientos del terreno, se utilizaron los siete movimientos de banda angosta incluidos en la tabla 5, los cuales se registraron en terrenos con periodos dominantes $\left(T_{g}\right)$ cercanos a los 2 segundos. Los movimientos se escalaron de tal manera que la velocidad máxima del terreno fuera igual a la correspondiente al registro SCT EO del sismo del 19 de septiembre de 1985.

En la figura 4 se muestran espectros de pseudo-aceleración para comportamiento elasto-plástico perfecto y ductilidades máximas $(\mu)$ de 2,3 y 4, los cuales corresponden a la media más una desviación estándar $(\sigma)$ de los espectros obtenidos para los diferentes movimientos del terreno. Note que las ordenadas espectrales mostradas en la figura mencionada definen la resistencia lateral de edificios de ocupación estándar; y estas mismas ordenadas multiplicadas por 1.5, la correspondiente a edificios del Grupo A. 
Tabla 5. Registros considerados para terreno blando

\begin{tabular}{cccc}
\hline Registro & Fecha & Componente & $T_{g}(\mathrm{seg})$ \\
\hline SCT & $09 / 19 / 85$ & EO & 2.0 \\
Tlahuac & $09 / 19 / 85$ & EO & 2.1 \\
Tlahuac & $09 / 21 / 85$ & EO & 1.9 \\
Tlahuac & $09 / 21 / 85$ & NS & 2.0 \\
Alameda & $04 / 25 / 89$ & EO & 2.1 \\
Alameda & $04 / 25 / 89$ & NS & 2.1 \\
Garibaldi & $04 / 25 / 89$ & EO & 2.2 \\
\hline
\end{tabular}

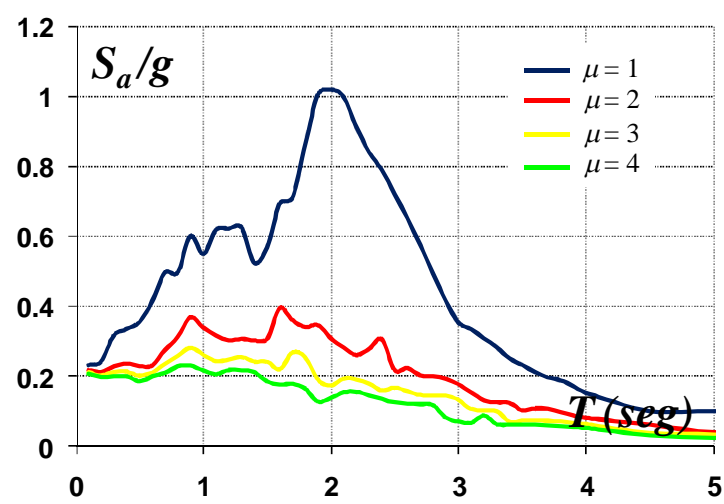

Figura 4. Espectros de resistencia para movimientos registrados en terreno blando

\section{Evaluación del desempeño de sistemas esenciales}

Para evaluar el desempeño de sistemas esenciales, se consideran edificios estructurados con base en marcos rígidos regulares. Para ello, se relaciona el número de pisos $(N)$ en los marcos con su periodo fundamental de vibración $(T)$ a través de la siguiente expresión ( $\beta$ es un coeficiente adimensional):

$T=\beta N$

Se consideraron edificios de 1 a 50 pisos y se supuso una altura de entrepiso de 4 metros. Con el fin de reducir el esfuerzo numérico involucrado en la estimación de su respuesta dinámica no lineal, se recurrió a modelar los edificios a través del sistema equivalente de un grado de libertad descrito con anterioridad. Note que una vez definido el número de pisos del edificio es posible establecer su periodo fundamental de vibración (ecuación 6) y su altura total. Mientras que para el valor del amortiguamiento se considero 5\% del crítico, se utilizo un valor del coeficiente de distorsión (COD) de 1.70 para $\mu \geq 2$ (ver tabla 3). Al parámetro $\beta$ se asignaron valores de $0.06,0.08$ y 0.10 y se consideró un comportamiento elasto-plástico perfecto para los sistemas equivalentes de un grado de libertad.

Para hacer posible un mejor entendimiento de las consecuencias de utilizar un factor de importancia de 1.5, se consideraron dos grupos de edificios. Mientras que ambos grupos exhiben las mismas propiedades en términos de periodos, alturas y coeficiente de amortiguamiento, las resistencias del segundo grupo (edificios esenciales) exhiben resistencias laterales que son $50 \%$ mayores respecto a las que corresponden al primer grupo (edificios con ocupación estándar). En cuanto a las resistencias del grupo que representa edificios de ocupación estándar, éstas corresponden exactamente a las ordenadas de los espectros mostrados en la figura 4 para ductilidades de 2,3 y 4. 
La figura 5 muestra demandas media $+\sigma$ de ductilidad máxima en sistemas estándar y esenciales. Mientras que $\mu$ denota la ductilidad máxima asociada al espectro utilizado para el diseño de los edificios, $\mu_{\max }$ denota la demanda máxima de ductilidad que desarrollan los mismos durante la excitación sísmica. Conforme a lo esperado, la figura 5a muestra que las demandas de ductilidad en los sistemas con ocupación estándar oscilan alrededor de 2, 3 y 4 . En cuanto a los sistemas esenciales, el impacto del factor de importancia no es igual en todo el rango de periodos. En particular, la demanda de ductilidad deja de ser constante con respecto al periodo y tiende a incrementarse desde un valor cercano a 0.8 para un periodo de cero, hasta valores cercanos a 1.3, 2.0 y 2.7 para el periodo dominante de las excitaciones ( 2 segundos) y ductilidades de diseño de 2,3 y 4, respectivamente. Note que el valor de la demanda de ductilidad tiende a permanecer razonablemente constante para sistemas cuyo periodo sea igual o mayor que el del terreno, y que los valores de $1.3,2.0$ y 2.7 se obtienen al normalizar el valor de las ductilidades de diseño (2, 3 y 4, respectivamente), por el valor del factor de importancia. En una primera aproximación al problema, puede decirse que el impacto del factor de importancia no es el mismo para todos los sistemas bajo consideración.
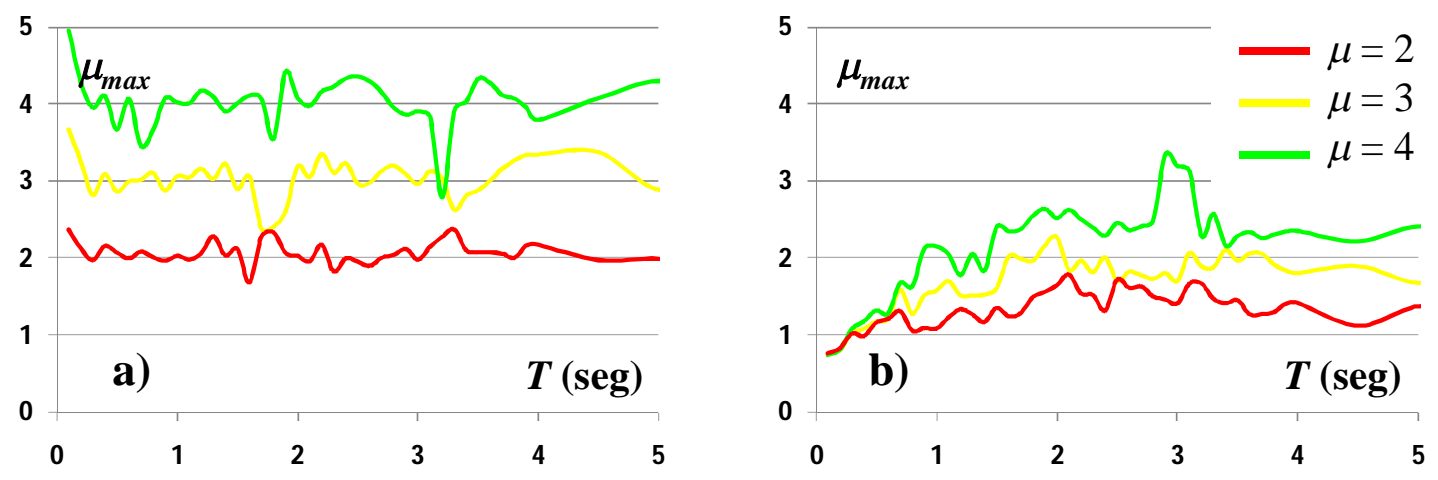

Figura 5. Demandas de ductilidad para sistemas sujetos a excitaciones registradas en suelo blando: a) Ocupación estándar; b) Estructuras esenciales

A través de la figura 6, que presenta demandas de distorsión, es posible discutir el desempeño estructural y no estructural de los edificios esenciales, y el impacto del factor de importancia. En términos del desempeño no estructural, es interesante notar que la demanda máxima de distorsión varía de manera notoria con el periodo, y que la diferente resistencia de los sistemas, ya sea porque fueron diseñados para diferente valor de $\mu$ o porque su diseño contempló el factor de importancia, no tiene un impacto importante en dicha demanda. En general e independientemente del valor de $\mu$ o de si la estructura es estándar o esencial, la distorsión para $\beta=0.10$ exhibe un valor muy bajo para un periodo de cero, y se incrementa con una tendencia lineal hasta alcanzar un valor ligeramente mayor que 0.015 para un periodo cercano al dominante del terreno ( 2 segundos). Una vez que se alcanza el valor pico de distorsión, ésta se reduce con un nuevo incremento de periodo, hasta que se estabiliza en un valor cercano a 0.005 para periodos alrededor de 5 segundos.

Dado lo anterior, es posible distinguir un rango de periodos para edificaciones de mediana altura (de 1 a 4 segundos) donde las demandas de distorsión exceden el umbral de 0.01, lo que implica daño de consideración en sistemas no estructurales adosados al sistema estructural, o la necesidad de usar un detallado especial en los elementos no estructurales para desconectarlos del sistema estructural. Puede verse también que el daño no estructural podría evitarse rigidizando de manera importante la estructura ( $\beta=0.06$, lo que implicaría aumentar alrededor de tres veces la rigidez del sistema con respecto al caso $\beta=0.10)$. Lo anterior indica que un gran porcentaje de edificios, ya sean esenciales o no, ubicados en la 
Zona del Lago del Distrito Federal requiere de consideraciones especiales en cuanto al detallado y conexión de los elementos no estructurales al sistema estructural.

El desempeño estructural puede evaluarse a partir de las demandas de distorsión plástica de entrepiso $\left(D I_{\max }^{p l}\right.$ ). Por ejemplo, considere que para vigas de concreto reforzado con buen detallado sísmico, umbrales de rotación plástica de $0.005,0.015$ y 0.025 pueden asociarse, respectivamente, a sus niveles de desempeño de Ocupación Inmediata, Seguridad de Vida, y Prevención de Colapso. Si se considera un valor de 0.002 para $\Delta \theta_{\max }$, entonces los umbrales de distorsión plástica de entrepiso para dichos niveles de desempeño deben ser, de acuerdo a la ecuación 5, de 0.003, 0.013 y 0.023, respectivamente.

Aunque la demanda de distorsión plástica varía de manera notoria con el periodo de los sistemas, la diferente resistencia de los sistemas ahora si se ve reflejada en dicha demanda. En general, independientemente del valor de $\mu$ o de si la estructura es estándar o esencial, la distorsión plástica exhibe un valor muy bajo para un periodo de cero, y se incrementa con una tendencia lineal hasta alcanzar un valor pico para un periodo cercano al dominante del terreno (2 segundos). Una vez que se alcanza el valor pico de distorsión, ésta se reduce con un nuevo incremento de periodo, hasta que se estabiliza para periodos alrededor de 5 segundos. En términos del efecto de la resistencia en sistemas de ocupación estándar con $\beta=0.10$, mientras que el valor pico de distorsión plástica alcanza valores cercanos a 0.01 para $\mu$ de 2 , este valor se incrementa hasta 0.015 para $\mu$ de 4 . El efecto del factor de importancia es notorio, ya que dichos valores picos se reducen a valores cercanos a 0.007 y 0.010 , respectivamente. De las curvas mostradas en la figura 6 es posible decir que el nivel de daño estructural esperado en los edificios es muy diferente, y va desde prácticamente nulo para sistemas con periodos muy cortos o muy grandes, hasta alcanzar niveles de importancia para edificios con periodos cercanos a los 2 segundos. A pesar de lo dicho, prácticamente ningún sistema de ocupación estándar excede el umbral de distorsión plástica de 0.013, asociado aquí con el nivel de desempeño denominado Seguridad de Vida. Lo anterior implica que todos los sistemas de ocupación estándar están en posición de cumplir con su principal objetivo de diseño, que es el de garantizar la seguridad de vida de los ocupantes de la edificación.

En contraste con lo anterior esta el caso de los sistemas que representan estructuras del Grupo A. Aunque el factor de importancia de 1.5 ha tenido un impacto en términos de reducir las demandas de comportamiento plástico en los sistemas estructurales, las distorsiones plásticas de varios sistemas exceden el umbral de 0.003 correspondiente a Ocupación Inmediata, aun cuando los sistemas cuenten con una mayor rigidez $(\beta=0.08$ y 0.06). Mientras que para $\mu$ de 2 existen algunos sistemas con periodos alrededor de 2 segundos que rebasan el umbral de Ocupación Inmediata, para $\mu$ de 4 y $\beta=0.10,0.08$ y 0.06 hay varios sistemas que rebasan de manera importante dicho umbral (con la consecuencia implícita de no garantizar la Ocupación Inmediata del edificio tras la ocurrencia del sismo de diseño).

\section{Espectros de rotación plástica constante}

El modelo equivalente de un grado de libertad no solo puede utilizarse para estimar la respuesta de edificios, sino para estimar la resistencia lateral que deben desarrollar para garantizar un desempeño estructural adecuado (en términos de garantizar su Ocupación Inmediata), y estudiar las demandas de ductilidad y distorsión de entrepiso asociadas a dicha resistencia. La figura 7 muestra espectros de resistencia, ductilidad y distorsión de entrepiso correspondientes a una distorsión plástica de entrepiso de 0.003. Los espectros corresponden a la media $+\sigma$ de los espectros correspondientes a cada movimiento del terreno. Conceptualmente, esta distorsión corresponde a una rotación plástica máxima en las vigas de los edificios de 0.005 , lo que implicaría que todos los edificios esenciales representados en dichos espectros queden en Ocupación Inmediata después de la excitación sísmica de diseño. 

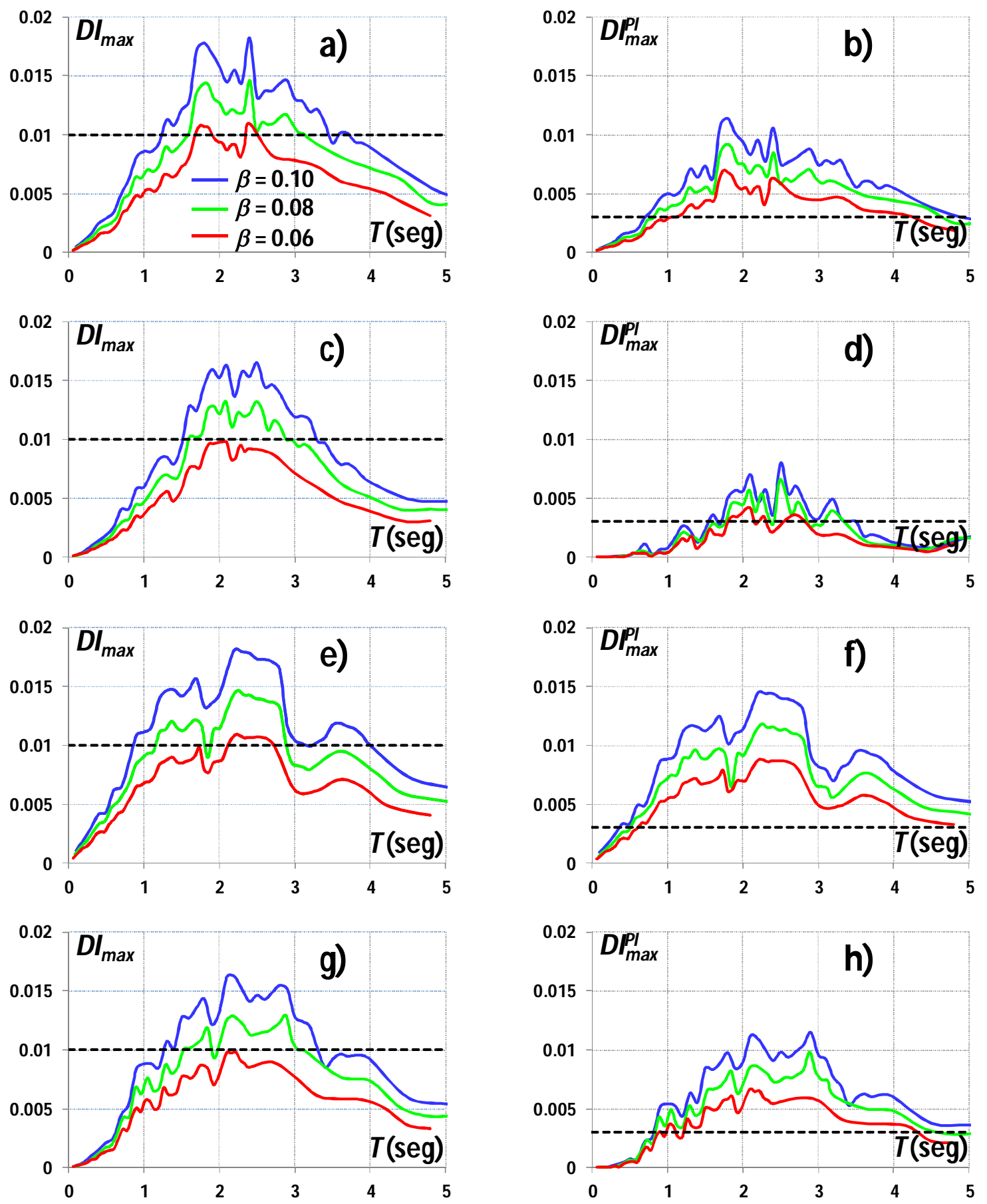

Figura 6. Demandas de $D I_{\max }$ y $D I_{\text {max }}^{p l}$ para sistemas sujetos a registros de terreno blando: a,b) Estándar, $\mu=2$; c,d) Esenciales, $\mu=2$; e,f) Estándar, $\mu=4$; g,h) Esenciales, $\mu=4$

Mientras que la figura 7a muestra con una línea roja la resistencia que deben alcanzar los sistemas esenciales para mantenerse en Ocupación Inmediata después de la excitación sísmica de diseño para un amortiguamiento del 5\% del crítico, la figura 8 muestra los factores de importancia que según las 
resistencias mostradas en la figura 7a requerirían ser usados para establecer la resistencia que deben desarrollar las estructuras del Grupo A si se usaran los espectros de resistencia correspondientes a ductilidades de 2, 3 y 4 y $\xi$ de $5 \%$. Es notoria la dependencia del factor de importancia con el periodo del sistema estructural y la ductilidad del espectro de resistencia. Además, es posible concluir que el valor de 1.5 utilizado actualmente para el factor de importancia resulta insuficiente para ductilidades de 3 y 4 . En caso de una ductilidad de 2 , el valor de 1.5 resulta escaso en un rango de periodos que va desde aproximadamente 1.5 segundos hasta 3 segundos.
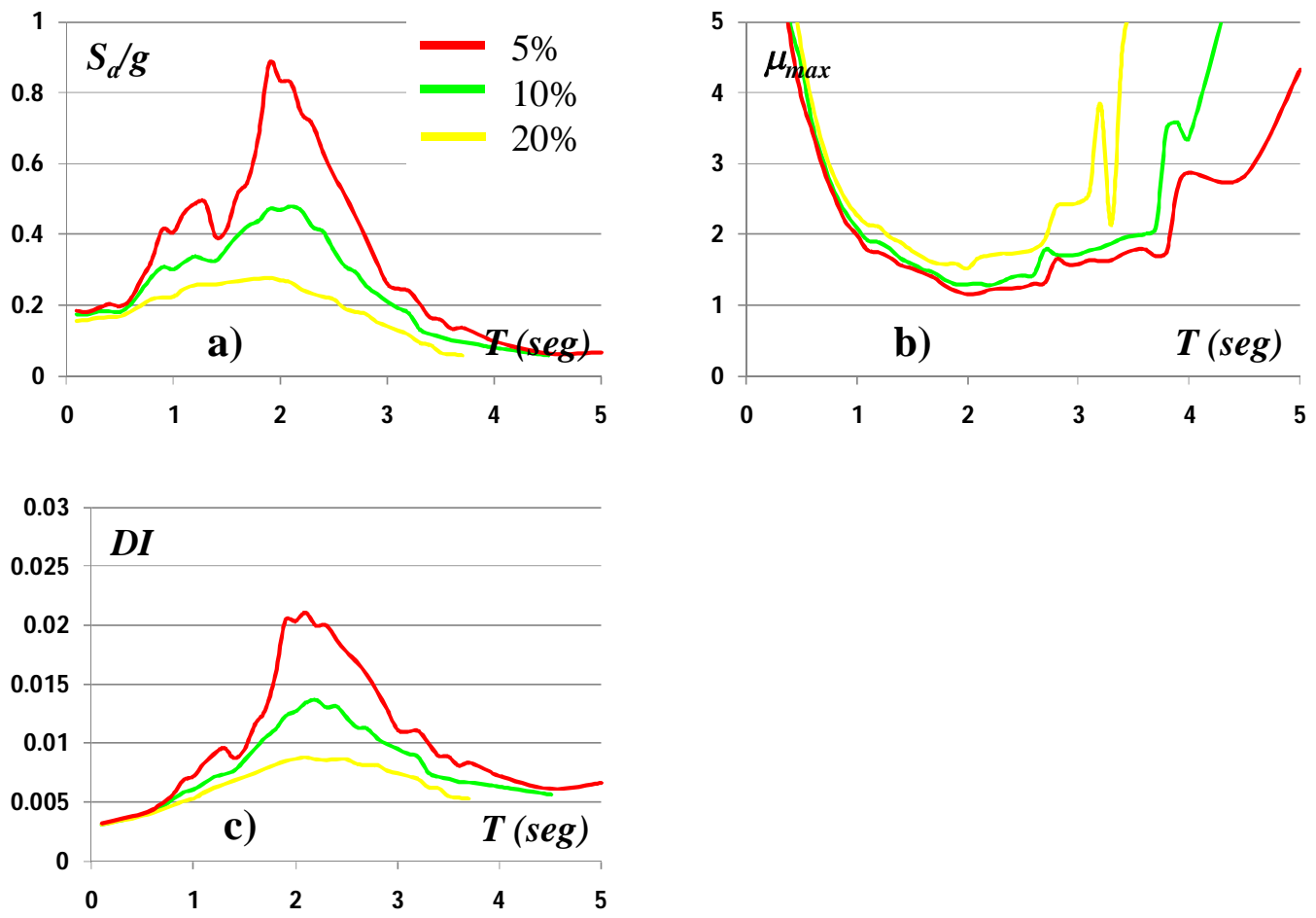

Figura 7. Espectros para Ocupación Inmediata $\left(D I_{\max }^{p l}=0.003\right)$ : a) Pseudo-aceleración; b) Demanda máxima de ductilidad; c) Distorsión de entrepiso

De lo anterior es posible establecer dos conclusiones preliminares: 1) Los sistemas esenciales desplantados en terreno blando no deben diseñarse con espectros de resistencia correspondientes a ductilidades mayores que 2; y 2) Aún si se utiliza un espectro de resistencia asociado a una ductilidad de 2 , el factor de importancia asociado a algunos edificios esenciales (particularmente aquellos cuyo periodo fundamental de vibración se acerque al valor de periodo dominante del terreno) puede llegar a ser sustancialmente mayor que 1.5 .

\section{METODOLOGÍA DE DISEÑO SISMORRESISTENTE BASADA EN DESPLAZAMIENTOS}

Dado que el uso de un factor de importancia durante el diseño sismorresistente de estructuras esenciales desplantadas en terreno blando muestra limitaciones, se propone en este estudio el desarrollo de herramientas de diseño basadas en desplazamientos. En particular, se presenta una metodología basada en desplazamientos para la concepción y diseño preliminar de estructuras esenciales, y que es capaz de resultar en sistemas estructurales que controlen de manera simultánea su daño estructural y no estructural a través del control apropiado de las demandas máximas de rotación plástica y de distorsión de entrepiso. 


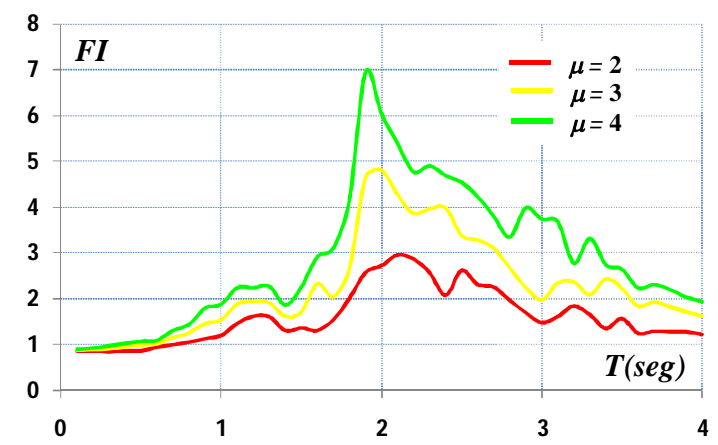

Figura 8. Factores de importancia requeridos para mantener a los sistemas esenciales en Ocupación Inmediata

\section{Descripción de la metodología}

La metodología, mostrada esquemáticamente en la figura 9, es aplicable al diseño de estructuras esenciales conformadas por marcos rígidos que exhiben, tanto en planta como en elevación, regularidad en términos de su geometría, resistencia, rigidez y masa; y considera que las estructuras deben cumplir con el nivel de desempeño de Ocupación Inmediata después de ocurrido un evento sísmico. En un contexto cualitativo podría decirse que el nivel Ocupación Inmediata se cumple cuando después de la ocurrencia del sismo los elementos estructurales muestran daño leve y no se presenta daño no estructural. Cuantitativamente, para asegurar un comportamiento estructural y no estructural adecuados, la metodología de diseño requiere que las demandas máximas de rotación plástica $\left(\theta_{p}^{\max }\right)$ y de distorsión de entrepiso $\left(D I_{\max }\right)$ queden por debajo de límites prestablecidos ( $\theta_{p}^{O I}$ y $D I_{N E}^{O I}$, respectivamente) de acuerdo al desempeño deseado.

El proceso de diseño empieza con la definición del sistema estructural y su configuración geométrica. En esta primera etapa se define también el material estructural y el tipo de elementos no estructurales. En términos del sistema estructural y no estructural elegidos, se establecen los límites aceptables de rotación plástica y distorsión máxima de entrepiso para cumplir con el nivel de desempeño de Ocupación Inmediata ( $\theta_{p}^{O I}$ y $D I_{N E}^{O I}$, respectivamente). De acuerdo a estos límites se establecen los umbrales de diseño para $\theta_{p}^{\max }$ y $D I_{\max }$, respectivamente. Una vez que el valor de $\theta_{p}^{\max }$ queda establecido, puede establecerse un valor límite para $D I_{\max }^{p l}$ mediante la ecuación 5.

Dado que el diseño basado en espectros de resistencia de ductilidad constante puede conducir a un desempeño sísmico inadecuado, para fines de la metodología se definen espectros de distorsión plástica máxima ( $D I_{\max }^{p l}$ ) constante. Dentro de este contexto, la relación establecida por el modelo simple de un grado de libertad entre $D I_{\max }^{p l}$ y las demandas de desplazamiento (ecuaciones 1 a 5 ) requiere de la disponibilidad de un valor de $\beta$, de tal manera que la metodología requiere de la suposición de un valor inicial para este parámetro (por ejemplo 0.10). Con el valor de $\beta$ puede estimarse el periodo fundamental de vibración de los marcos mediante la ecuación 6.

El valor de $D I_{\max }$ puede usarse para establecer el umbral de diseño para el desplazamiento lateral máximo de azotea (el significado de todas las variables ha sido definido con anterioridad):

$$
\delta_{\max }=\frac{D I_{\max } H}{C O D}
$$


Con base en las demandas de ductilidad estimadas en la figura $7 \mathrm{~b}$ y los valores de $\alpha$ y $C O D$ mostrados en las tablas 2 y 3 , la figura 10 muestra ayudas de diseño para calcular los valores de $C O D$ y $\alpha$ necesarios para la aplicación de la metodología propuesta. La figura 10a muestra la variación de COD como función del periodo de los marcos a diseñar.

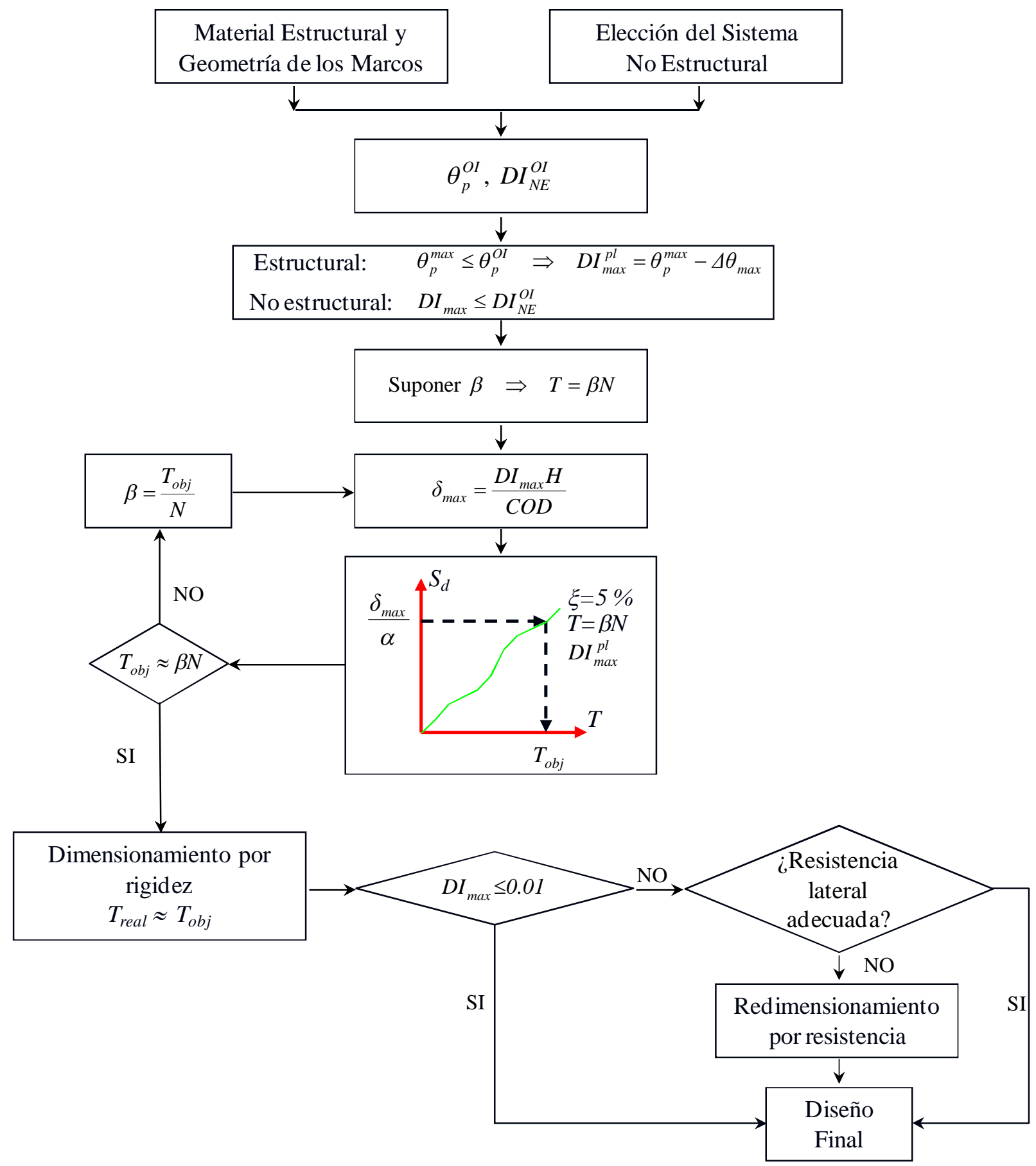

Figura 9. Metodología de diseño basada en desplazamientos 

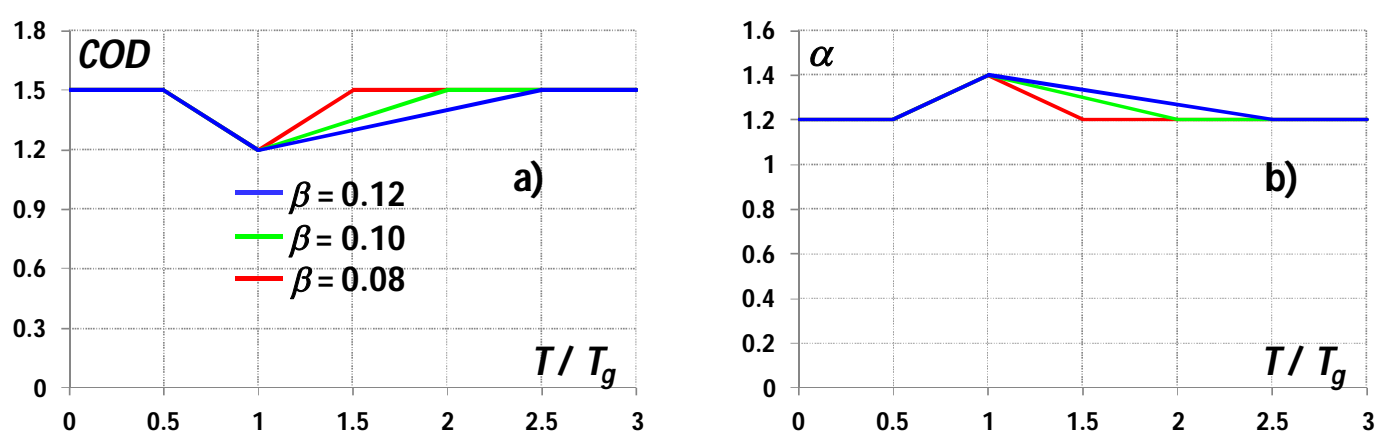

Figura 10. Valores recomendados para parámetros utilizados en el diseño de marcos de estructuras esenciales de cinco o más niveles $\left(N=5+, \theta_{p}^{\max }=0.005, \xi=0.05\right)$ : a) $C O D$; b) $\alpha$

El periodo fundamental de vibración objetivo $\left(T_{o b j}\right)$ de los marcos por diseñar puede estimarse a través de un espectro de desplazamientos para $D I_{\max }^{p l}$ constante (figura 11) y el valor normalizado de $\delta_{\max }$. En particular, $\delta_{\max }$ debe normalizarse por $\alpha$ para tomar en consideración los efectos de múltiples grados de libertad. El valor del parámetro $\alpha$ puede calcularse con la ayuda de diseño mostrada en la figura 10b, la cual se formula por medio de plantear consideraciones similares a las usadas para establecer la figura 10a. Observe que el espectro de desplazamientos de diseño corresponde a valores específicos de $D I_{\max }^{p l}$ y $\beta, \mathrm{y}$ que un 5\% de amortiguamiento crítico representa un límite inferior razonable para materiales estructurales que alcanzan o exceden su esfuerzo de fluencia (Chopra, 2001).

Una vez que se estima $T_{o b j}$, debe revisarse que su valor sea congruente con el valor planteado inicialmente para $\beta$. Si $T_{o b j}$ es aproximadamente igual a $\beta N$, se procede a dimensionar los elementos estructurales de tal manera que el periodo fundamental de vibración de la estructura sea similar a $T_{o b j}$. En caso contrario, se actualiza el valor de $\beta$ y se itera. Dentro de este contexto, es importante enfatizar que $\delta_{\max }$ es un dato establecido en función de los niveles aceptables de daño, y $T_{o b j}$ una parámetro que debe establecerse con el fin cuantificar a nivel global los requerimientos de rigidez lateral. Note que los valores de $\beta$ y $T_{o b j}$ no se conocen de antemano y que son mutuamente dependientes. Debido a esto, el procedimiento propuesto requiere en ocasiones de iteraciones de diseño.

En esta etapa de diseño se considera que las dimensiones de vigas y columnas son adecuadas si proporcionan a la estructura la rigidez necesaria para que el periodo de la estructura sea semejante a $T_{o b j}$. Una vez que las vigas y columnas de los marcos son dimensionadas, la metodología procede a su etapa final si los umbrales de diseño propuestos para $D I_{\max }$ no exceden 0.01. El diseño final consiste en dos tareas: a) la verificación del diseño preliminar mediante una serie de análisis dinámicos no lineales; y b) el ajuste del tamaño de vigas y columnas de tal manera que los marcos diseñados cumplan adecuadamente con el nivel de desempeño de Ocupación Inmediata. El uso de la metodología aquí presentada para el diseño preliminar de marcos que conforman estructuras esenciales, lleva a la versión final de la estructura diseñada con pocas o ninguna iteración.

En el caso que $D I_{\max }$ sea mayor que 0.01 , se recomienda revisar la resistencia lateral del marco con respecto a la requerida de acuerdo al espectro de resistencia de diseño. En este sentido, el coeficiente sísmico estimado para los marcos a partir de un análisis estático no lineal debe ser adecuado cuando se le compara con la ordenada del espectro de resistencia correspondiente al periodo real de los marcos. En el caso de que la resistencia lateral de los marcos sea insuficiente, las vigas y las columnas deben ser rediseñadas para corregir las deficiencias encontradas. Esta revisión será ilustrada en los ejemplos desarrollados en la siguiente sección. 

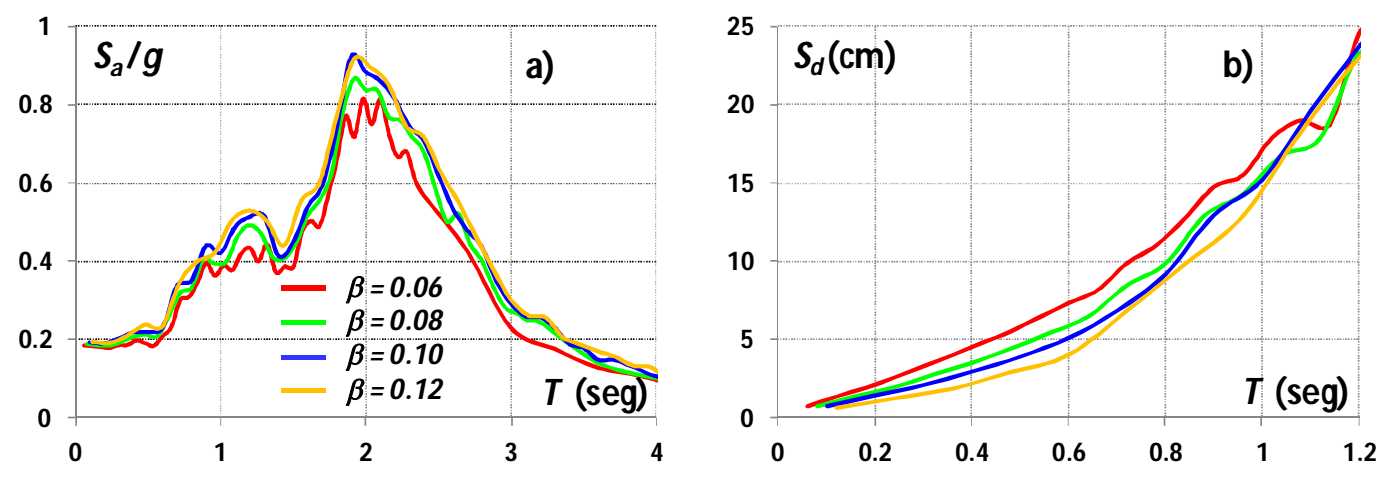

Figura 11. Espectros de diseño para $D I_{\max }^{p l}=0.003$ y $\xi=0.05$ : a) Resistencia; b) Desplazamiento

\section{EJEMPLOS DE DISEÑO}

Para ilustrar la aplicación de la metodología se diseñan dos versiones de un sistema estructurado con base en marcos rígidos de acero (Modelo 1 y Modelo 2) y desplantado en la Zona del Lago del Distrito Federal. Los dos modelos comparten tanto la configuración como el material estructural, y se diferencian en términos de la cuantificación que se hace de sus objetivos de diseño. En particular, mientras que la primera versión se diseña para una $D I_{N E}^{O I}$ de 0.007 , la segunda versión considera un valor límite de 0.01. En términos del desempeño estructural, ambos modelos son diseñados para no exceder una rotación plástica $\theta_{p}^{o I}$ de 0.005 . Además, los marcos fueron diseñados conforme al enfoque de diseño por capacidad para que desarrollen mecanismos de viga débil-columna fuerte.

Los marcos por diseñar conforman un edificio de ocho niveles con altura de entrepiso de 3 metros, y con planta simétrica de $18 \mathrm{~m} \times 18 \mathrm{~m}$. Los marcos de acero se construyen con vigas estructuradas con perfiles W y columnas de sección cajón cuadradas. La figura 12 esquematiza la planta y elevación de los edificios. Vale la pena mencionar que el diseño contempla tres cambios de secciones en altura, ya que se conservan las mismas secciones en los niveles 1-3, 4-6 y 7-8.

Si se supone que $\Delta \theta_{\max }=0.002$, de la ecuación $5 D I_{\max }^{p l}=0.003$ para $\theta_{p}^{\max }$ de 0.005 . Dado que la rotación plástica máxima de diseño es la misma, ambas versiones del edificio requieren que se formule el espectro de desplazamientos de diseño para una $D I_{\text {max }}^{p l}$ de 0.003 . La figura 11 muestra espectros de diseño de resistencia y desplazamiento correspondientes a diferentes valores de $\beta$. Los espectros se establecieron a partir de la media $+\sigma$ de los valores correspondientes a cada uno de los registros incluidos en la tabla 5 .

En una primera iteración se consideró $\beta=0.10$, de tal manera que el periodo fundamental de vibración estimado inicialmente para ambos modelos fue de $0.10 \times 8=0.8$ segundos. Dado que $T_{g}=2.0$ segundos, $T / T_{g}=0.4$. De las ayudas de diseño de la figura 10 , se asignan valores de 1.5 y 1.2 para $C O D$ y $\alpha$, respectivamente. De acuerdo con la ecuación 7, $\delta_{\max }$ adopta valores de 11.2 y $16.0 \mathrm{~cm}$, respectivamente, para el Modelo $1 \mathrm{y}$ el Modelo 2 (note que las distorsiones de diseño no son las mismas para ambas versiones del edificio y por ello los diferentes umbrales para el desplazamiento de azotea). Estos últimos valores llevaron a valores normalizados $\delta_{\max } / \alpha$ de 9.3 y 13.3. Conforme al diagrama de flujo de la figura 9 , se obtiene un $T_{o b j}$ de 0.8 segundos para el Modelo $1 \mathrm{y}$, después de una sola iteración, un valor de 0.96 
segundos para el segundo modelo (para este último caso el valor final de $\beta$ resultó ser 0.12 ). La figura 13 muestra la manera como se estima el valor de $T_{o b j}$ para las dos versiones del edificio.
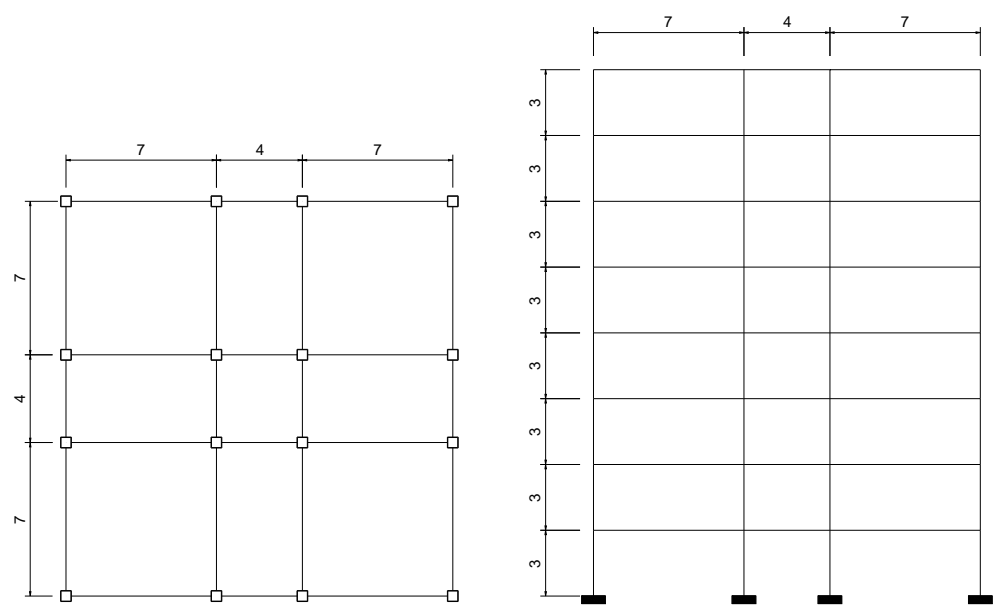

Figura 12. Vista en planta y elevación de la estructura de ocho niveles (dimensiones en metros)
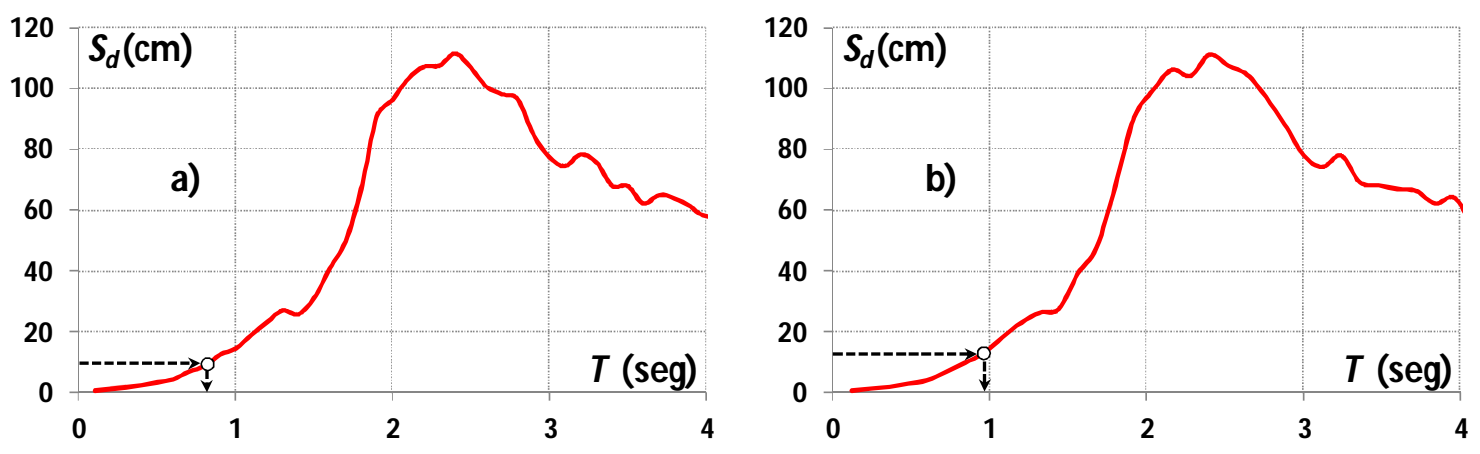

Figura 13. Determinación de $T_{o b j}$ : a) Modelo 1 ( $\left.\beta=0.10\right)$; b) Modelo $2(\beta=0.12)$

Conforme al diagrama de flujo de la figura 9, deben dimensionarse lo elementos estructurales de manera que el periodo fundamental de los marcos sea lo suficientemente cercano al valor de $T_{o b j}$. La tabla 6 resume las dimensiones de vigas y columnas para las dos versiones del edificio. Mientras que para el Modelo 1 se obtiene un $T_{\text {real }}$ de 0.81 segundos, para el Modelo 2 se obtuvo un periodo de 0.97 segundos. En ambos casos, se consideró acero A36 para los elementos estructurales de los marcos.

Una vez que se dispuso de las dimensiones de vigas y columnas, se prepararon modelos para llevar a cabo una serie de análisis no lineales con el programa DRAIN 2Dx (Prakash et al., 1993) para evaluar la respuesta sísmica de las dos versiones del edificio. Para ello, se modelaron dos marcos de los cuatro que conforman la estructura completa con sus propiedades de rigidez y resistencia duplicadas. Dada la regularidad en planta de la estructura es posible estimar de manera razonable su respuesta dinámica a través de un modelo plano. Mientras que las vigas de los modelos contemplan un comportamiento bilineal con $2 \%$ de endurecimiento por deformación, el modelo de las columnas considera el efecto combinado de la carga axial y el momento flexionante, y un comportamiento bilineal sin endurecimiento por deformación. La resistencia nominal del acero se incrementó en $20 \%$ para que reflejara el esfuerzo de fluencia esperado en el campo (Wong, 2009). Se consideraron los efectos $P$ - $\Delta$ mediante el planteamiento de una matriz de rigidez geométrica y se supuso que las bases de las columnas de la planta baja están empotradas. Para el caso de los análisis dinámicos no lineales, se consideró un amortiguamiento del 5\% 
del crítico para los dos primeros modos de vibrar a través de una matriz de Rayleigh. La figura 14 muestra la media $+\sigma$ de las demandas en altura de distorsión máxima de entrepiso y de rotación plástica máxima para los dos modelos del edificio. Ambos modelos son capaces de controlar adecuadamente las demandas de $D I_{\text {max }}$ dentro de los límites de diseño.

Tabla 6. Secciones transversales de vigas y columnas de los dos modelos del edificio diseñado

\begin{tabular}{ccccccc}
\hline & \multicolumn{2}{c}{ Modelo 1 } & \multicolumn{3}{c}{ Modelo 2 } \\
\cline { 2 - 6 } Niveles & \multicolumn{2}{c}{ Columnas } & Vigas & \multicolumn{2}{c}{ Columnas } & Vigas \\
\cline { 2 - 7 } & Lado (cm) $\begin{array}{c}\text { Espesor de } \\
\text { placa }(\mathrm{cm})\end{array}$ & Sección Lado (cm) $\begin{array}{c}\text { Espesor de } \\
\text { placa (cm) }\end{array}$ & Sección \\
\hline $1-3$ & 70 & 3.175 & W18X97 & 60 & 2.540 & W18X86 \\
$4-6$ & 65 & 2.540 & W18X76 & 55 & 1.905 & W18X76 \\
$7-8$ & 60 & 2.540 & W18X71 & 50 & 1.905 & W18X60 \\
\hline
\end{tabular}

En términos de su desempeño estructural, los dos modelos también cumplen con sus objetivos de diseño. Por un lado, note que las demandas de $\theta_{p}^{\max }$ mostradas en la figura $14 \mathrm{~d}$ están cerca de su límite de diseño de 0.005, y que estas son mayores que aquellas presentadas en la figura 14b. Por el otro lado, mientras que las demandas de $\theta_{p}^{\max }$ mostradas en la figura 14b muestran la baja probabilidad de que los elementos estructurales de los marcos diseñados para $D I_{\max } \leq 0.01$ tengan que ser redimensionados en términos de su resistencia; las demandas de $\theta_{p}^{\max }$ mostradas en la figura $14 \mathrm{~d}$ reflejan la importancia de revisar la resistencia lateral de marcos diseñados para $D I_{\max }>0.01$. Lo anterior confirma e ilustra el hecho de que si la distorsión permisible en los marcos diseñados crece, el dimensionado de vigas y columnas puede ser gobernado por resistencia y no por rigidez, como sucede en el caso en que las distorsiones de diseño sean menores que 0.01 .
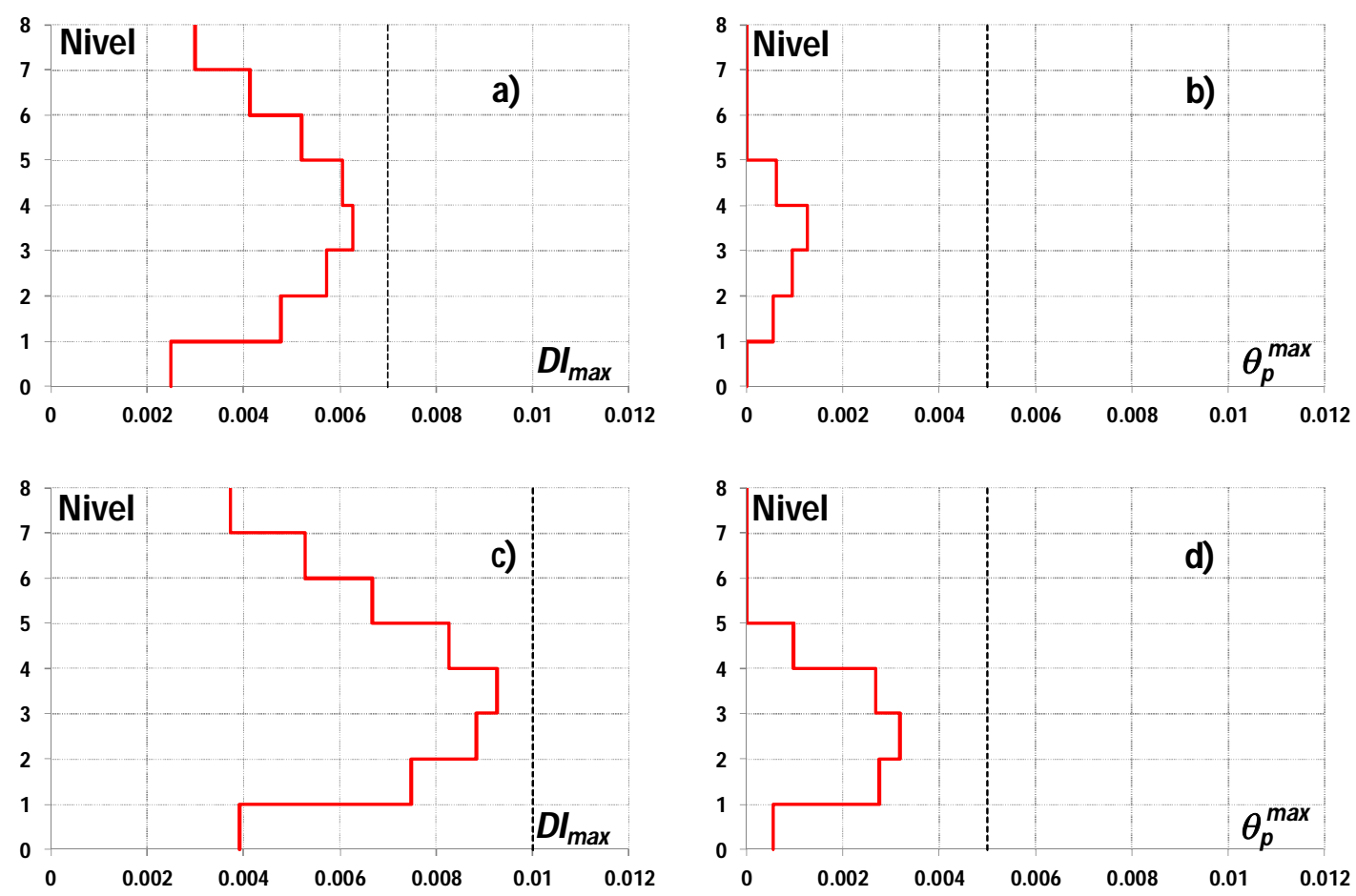

Figura 14. Demandas máximas de deformación en altura para ambas versiones del edificio:

a) $D I_{\max }$, Modelo 1; b) $\theta_{p}^{\max }$, Modelo 1; c) $D I_{\max }$, Modelo 2; d) $\theta_{p}^{\max }$, Modelo 2 
En cuanto a la metodología de diseño propuesta, puede decirse que su aplicación a los dos modelos presentados resultó en diseños que cumplieron adecuadamente con el nivel de desempeño de Ocupación Inmediata. Aún así y sólo para ilustrar la revisión por resistencia que se requiere para los marcos diseñados para $D I_{\max }>0.01$, la figura 15 muestra las curvas de capacidad y los espectros de resistencia de diseño para los dos modelos del edificio. En términos de la resistencia lateral requerida, las figuras 15a y $15 \mathrm{c}$ muestran coeficientes sísmicos de diseño de 0.36 y 0.43 respectivamente. En términos del coeficiente sísmico real de los marcos, la idealización bilineal de las curvas de capacidad mostradas en las figuras $15 \mathrm{~b}$ y $15 \mathrm{~d}$ muestran coeficientes sísmicos a la fluencia de alrededor de 0.55 and 0.42 , respectivamente.

Si se considera que la respuesta de los edificios está totalmente dominada por el primer modo de vibrar y que este primer modo excita alrededor del $85 \%$ del total de la masa de los marcos, las demandas de resistencia en términos del coeficiente sísmico se pueden expresar como $0.85 \times 0.36=0.31$ y $0.85 \times$ $0.43=0.37$, respectivamente. Mientras que el Modelo 1 muestra una resistencia significativamente más grande respecto a su demanda (0.55 vs 0.31), el Modelo 2, diseñado para $D I_{\max }=0.01$, muestra una relación más balanceada $(0.42$ vs 0.37$)$.
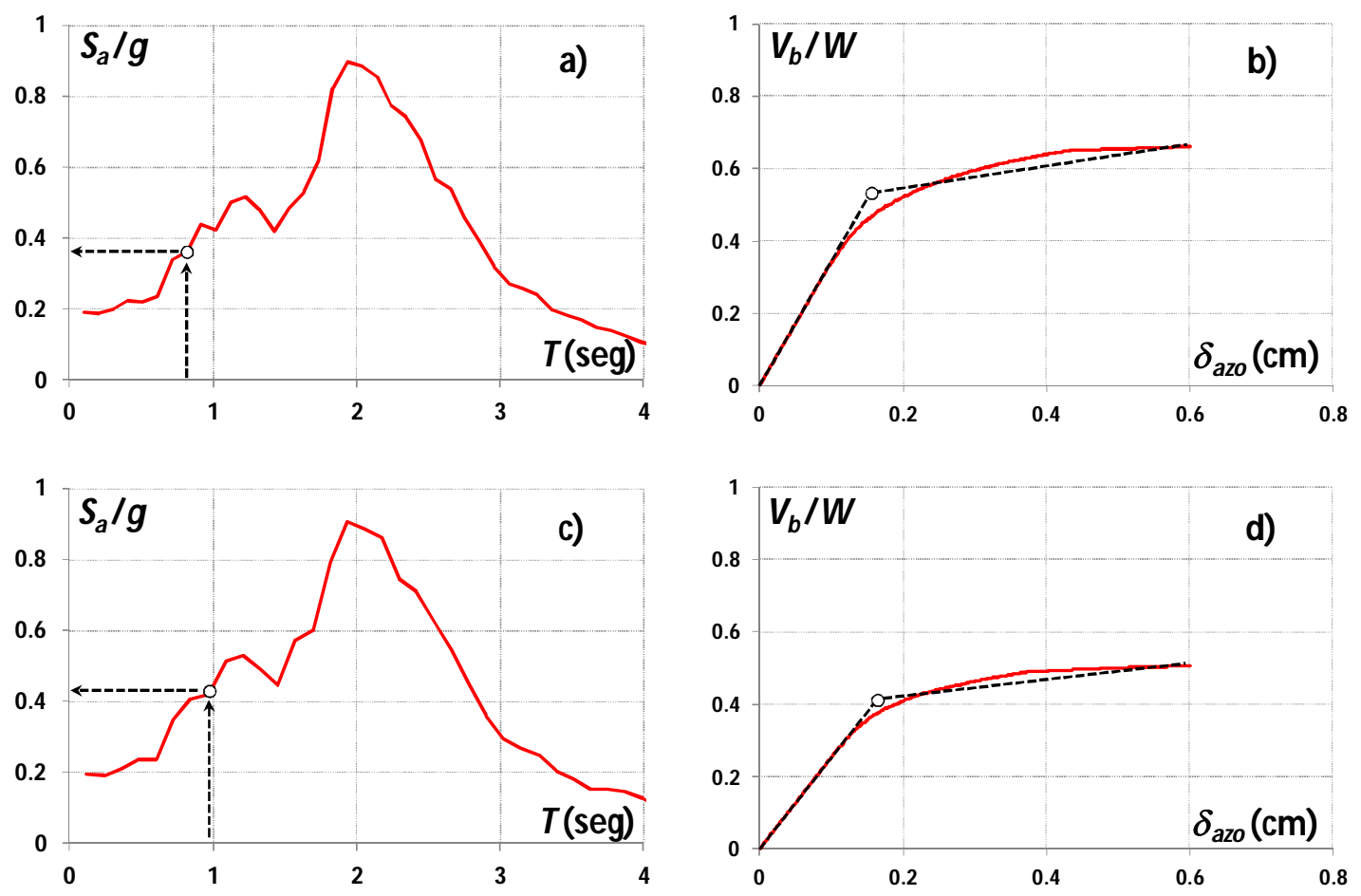

Figura 15. Revisión por resistencia $\xi=0.05$ y $D I_{\max }^{P}=0.003$ : a) Demanda de resistencia $(\beta=0.10)$ y; b) Curva de capacidad, Modelo 1; c) Demanda de resistencia $(\beta=0.12)$ y; d) Curva de capacidad, Modelo 2

\section{Versiones diseñadas de acuerdo al Reglamento de Construcciones para el Distrito Federal}

Se diseñaron dos modelos más de la estructura bajo consideración, pero ahora de acuerdo a los requerimientos de diseño del Reglamento de Construcciones para el Distrito Federal (RCDF, 2004). Se optó por utilizar un factor de comportamiento sísmico de 2 y el espectro de diseño correspondiente a la Zona IIIb. Al tratarse de una estructura del Grupo A, las fuerzas de diseño se incrementaron por un factor de importancia de 1.5. Mientras que uno de los nuevos modelos se diseñó para una distorsión máxima de 
entrepiso de 0.006 (Modelo 3), el otro consideró un valor de 0.012 (Modelo 4). Ambos modelos consideraron el método dinámico (modal espectral) durante su diseño. La tabla 7 muestra comparaciones entre las dimensiones y peso de los elementos estructurales de los Modelos 1 y 3; y entre los valores correspondientes a los Modelos 2 y 4 . Note que la metodología propuesta conduce a diseños que resultan más ligeros que los hechos de acuerdo al reglamento. Se observan reducciones que van del 25 al $30 \%$ del peso del sistema estructural. Vale la pena mencionar que al analizar los modelos diseñados conforme a norma, se observa que ambos sistemas permanecen elásticos durante el sismo de diseño y son capaces de satisfacer el nivel de desempeño correspondiente a Ocupación Inmediata. Lo anterior corrobora lo observado en la figura 8 , donde se observa que para edificios de baja altura, el factor de importancia requerido para mantener los marcos en Ocupación Inmediata es menor que 1.5. La figura 16 muestra que los perfiles en altura de distorsión para los Modelos 3 y 4 implican demandas de deformación que son mucho menores que las implícitas en su diseño.
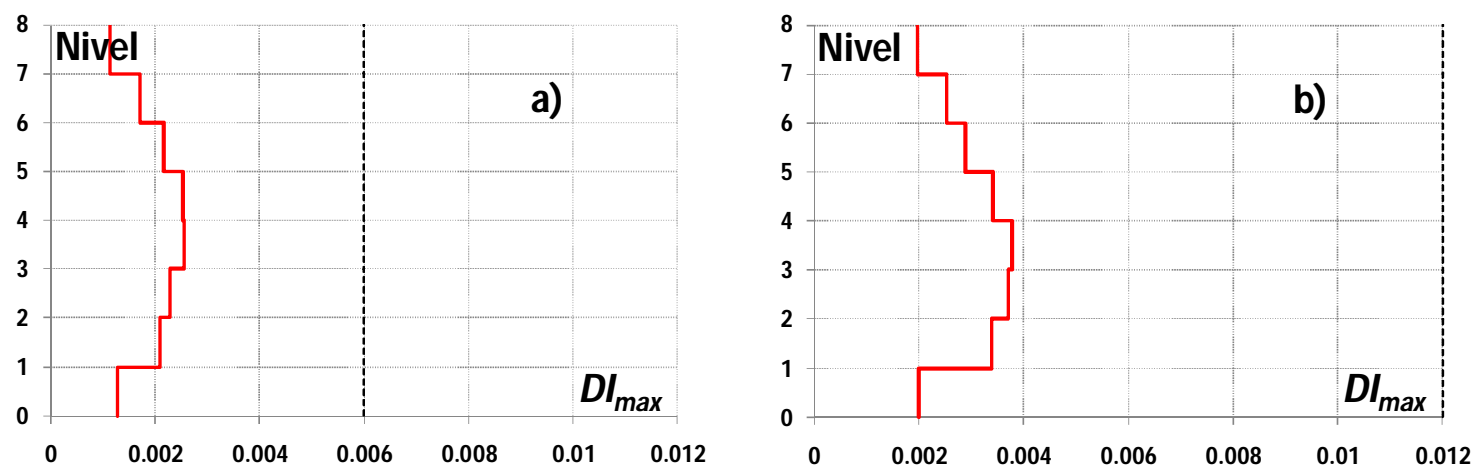

Figura 16. Demandas máximas de deformación en altura para versiones del edificio diseñadas de acuerdo al Reglamento de Construcciones para el Distrito Federal: a) DI $I_{\max }$, Modelo 3; b) DI $I_{\max }$, Modelo 4

Tabla 7. Comparación entre Modelos 1 y 3 y entre Modelos 2 y 4

\begin{tabular}{|c|c|c|c|c|}
\hline \multicolumn{4}{|c|}{ Modelo 1 Acero A36 } & \multirow[t]{2}{*}{$\mathrm{W}_{\text {Modelo } 1} / \mathrm{W}_{\text {Modelo } 3}$} \\
\hline $\mathrm{T}=0.81$ segundos & & Columnas & Vigas & \\
\hline Nivel & Lado $[\mathrm{cm}]$ & Espesor de placa [cm] & Sección & \\
\hline $1-3$ & 70 & 3.175 & W18X97 & \multirow{5}{*}{0.75} \\
\hline $4-6$ & 65 & 2.540 & W18X76 & \\
\hline $7-8$ & 60 & 2.540 & W18X71 & \\
\hline \multicolumn{4}{|c|}{ Modelo 3 Acero A36 } & \\
\hline $\mathrm{T}=0.57$ segundos & & Columnas & Vigas & \\
\hline Nivel & Lado $[\mathrm{cm}]$ & Espesor de placa $[\mathrm{cm}]$ & Sección & \\
\hline $1-3$ & 70 & 3.175 & W24X162 & \\
\hline $4-6$ & 65 & 2.540 & W21X132 & \\
\hline $7-8$ & 60 & 2.540 & W21X122 & \\
\hline \multicolumn{4}{|c|}{ Modelo 2 Acero A36 } & $\mathrm{W}_{\text {Modelo 2 }} / \mathrm{W}_{\text {Modelo 4 }}$ \\
\hline $\mathrm{T}=0.92$ segundos & & Columnas & Vigas & \\
\hline Nivel & Lado $[\mathrm{cm}]$ & Espesor de placa $[\mathrm{cm}]$ & Sección & \\
\hline $1-3$ & 60 & 2.540 & W18X86 & \multirow{5}{*}{0.71} \\
\hline $4-6$ & 55 & 1.905 & W18X76 & \\
\hline $7-8$ & 50 & 1.905 & W18X60 & \\
\hline \multicolumn{4}{|c|}{ Modelo 4 Acero A36 } & \\
\hline $\mathrm{T}=0.65$ segundos & & Columnas & Vigas & \\
\hline Nivel & Lado $[\mathrm{cm}]$ & Espesor de placa [cm] & Sección & \\
\hline $1-3$ & 65 & 2.540 & W21X132 & \\
\hline $4-6$ & 60 & 2.540 & W21X122 & \\
\hline $7-8$ & 55 & 2.540 & W18X86 & \\
\hline
\end{tabular}




\section{CONCLUSIONES Y COMENTARIOS FINALES}

Las estructuras catalogadas como esenciales son sistemas altamente complejos que son vulnerables a la acción destructiva de los sismos. Debido a lo refinado y complejo de su operación cotidiana, el diseño de las instalaciones esenciales requiere de la consideración cuidadosa del desempeño de todos sus subsistemas. Como consecuencia de los daños estructurales que han exhibido estas instalaciones durante sismos severos, la normatividad de varios países requiere del uso de un factor de importancia, generalmente igual a 1.5, para incrementar las fuerzas laterales de diseño.

De los resultados presentados, puede decirse que el desempeño estructural de sistemas esenciales en terreno blando depende de manera muy importante de su periodo, ya que mientras aquellos sistemas que exhiben un periodo cercano al periodo dominante del terreno exhiben un desempeño estructural deficiente, aquellos que se alejan de dicho periodo no requieren de consideraciones especiales en cuanto a su diseño de resistencia. En particular, los sistemas que exhiben un periodo similar al periodo dominante del terreno deben diseñarse para permanecer prácticamente elásticos durante la excitación sísmica de diseño para que sean capaces de controlar sus demandas de rotación plástica dentro de umbrales congruentes con el nivel de desempeño de Ocupación Inmediata.

Independientemente del detallado utilizado para los elementos estructurales de una estructura esencial, no es conveniente considerar ductilidades mayores que 2 durante su diseño por resistencia. Aunque puede ser deseable proporcionar detallado dúctil al sistema estructural de un sistema esencial, la necesidad de mantener su sistema estructural en Ocupación Inmediata después de la excitación sísmica de diseño puede implicar restringir de manera importante sus demandas plásticas de deformación. Bajo estas circunstancias, no es posible aprovechar la capacidad de deformación plástica del sistema estructural para reducir las fuerzas laterales de diseño.

En cuanto al desempeño no estructural de sistemas estándar y esenciales ubicados en terreno blando (diseñados estos últimos con un factor de importancia de 1.5, es decir, de acuerdo a la reglamentación vigente en el Distrito Federal), se estimaron distorsiones de entrepiso iguales o mayores que 0.01 para ambos tipos de estructuras (Grupos A y B) cuando el valor del periodo fundamental de vibración del sistema estudiado se acerca al valor de periodo dominante del terreno (ver figuras 6a, 6c, 6e y 6g). Bajo estas circunstancias el desempeño no estructural tenderá a ser deficiente, y solo llegará a ser satisfactorio si se usa un detallado especial, de tal manera de desconectar los elementos no estructurales del sistema estructural de la edificación. En lo referente a los sistemas esenciales ubicados en terreno blando y diseñados de acuerdo al RCDF (RCDF, 2004) y cuyo periodo se acerca al periodo dominante del terreno, mantenerlos prácticamente elásticos durante el sismo de diseño para garantizar un desempeño estructural adecuado (figura $7 \mathrm{~b}$ ) resulta en distorsiones cercanas a 0.02 (figura 7c), demanda que implicaría daños en sistemas no estructurales tradicionales, aún si estos fueran desconectados del sistema estructural y detallados para acomodar deformaciones laterales de importancia. Bajo estas circunstancias, la única manera de hacer posible de manera simultánea desempeños estructural y no estructural adecuados es el uso de sistemas disipadores de energía.

A partir del mal desempeño estimado para algunas estructuras esenciales diseñadas de acuerdo al Reglamento de Construcciones para el Distrito Federal, puede afirmarse que para lograr un desempeño adecuado de estructuras esenciales desplantadas en terreno blando como el de la Zona del Lago de Distrito Federal, es necesario un control explícito de su respuesta dinámica. Dentro de este contexto, metodologías simples basadas en desplazamientos pueden usarse para el diseño preliminar de marcos rígidos que sean capaces de controlar adecuadamente el daño estructural y no estructural en instalaciones esenciales mediante el control simultáneo de las demandas máximas de distorsión lateral y rotación plástica. El uso de la metodología propuesta en este trabajo resultó en el diseño de dos versiones de un sistema estructural 
que han sido capaces de cumplir con el nivel de desempeño de Ocupación Inmediata. Es importante que en la siguiente etapa del trabajo que aquí se presenta, se aplique la metodología a una estructura cuyo periodo fundamental de vibración se acerque más al periodo dominante del terreno, de tal manera que se considere el caso de un sistema estructural que desarrolle demandas importantes de deformación lateral. Además, será necesario ampliar el campo de aplicación de la metodología para que pueda utilizarse a otros materiales y configuraciones estructurales (particularmente en lo que se refiere a irregularidades estructurales en planta y altura).

Los planteamientos ofrecidos en este artículo han considerado un enfoque determinista. Dentro de este contexto, la manera en que se han agrupado y escalado los movimientos del terreno no contempla un marco estadístico formal. Bajo estas circunstancias y aunque los resultados que se presentaron aportan un entendimiento de lo que debe hacerse para lograr un diseño sísmico adecuado de estructuras esenciales ubicadas en la Zona del Lago del Distrito Federal, la elaboración de requerimientos normativos de diseño necesita plantear un marco que contemple explícitamente las incertidumbres involucradas en la determinación de las demandas y capacidades sísmicas, y la definición clara de: A) La tasa anual de excedencia asociada a los espectros de diseño; B) Las condiciones bajo las cuales se satisface el nivel de desempeño de Ocupación Inmediata desde puntos de vista estructural y no estructural; y C) La tasa anual de excedencia aceptable para dicho nivel de desempeño. Las conclusiones ofrecidas en términos del uso del factor de importancia para el diseño de la resistencia lateral de un sistema esencial, quedan referidas a una definición de resistencia lateral que contempla aquella que debe alcanzar la estructura esencial en su rango plástico de comportamiento cuando se le sujeta a la acción del sismo de diseño. Dado que las estructuras sismorresistentes tienden a exhibir una resistencia lateral mayor que la considerada durante el diseño, una propuesta normativa debe especificar resistencias laterales de diseño bajo la consideración de la llamada sobrerresistencia. Bajo estas circunstancias, la especificación de una resistencia lateral de diseño para una estructura esencial debe considerar que dicha sobrerresistencia puede exhibir valores diferentes a los que corresponden a estructuras de ocupación estándar.

\section{AGRADECIMIENTOS}

Los dos primeros autores agradecen el apoyo de la Universidad Autónoma Metropolitana, y quisieran dedicar este artículo a la memoria del tercer autor. El primer autor agradece al Consejo Nacional de Ciencia y Tecnología la beca otorgada para sus estudios de posgrado. Los dos primeros autores agradecen a los revisores del manuscrito sus atinados comentarios los cuales mejoraron y enriquecieron el trabajo final.

\section{REFERENCIAS}

Applied Technology Council (1998), "Evaluation of earthquake damaged concrete and masonry wall buildings, basic procedures manual", Reporte, FEMA 306.

Bertero, V V, J C Anderson, H Krawinkler y E Miranda, (1991), "Design guidelines for ductility and drift limits: review of state-of-the-practice and state-of-the-art in ductility and drift-based earthquakeresistant design of buildings", Reporte, UCB/EERC-91/15, University of California at Berkeley.

Bertero, R D y V V Bertero, (1992), "Tall reinforced concrete buildings: conceptual earthquake-resistant design methodology", Reporte, UCB/EERC-92/16, University of California at Berkeley. 
Chopra, A K (2001), Dynamics of structures, theory and applications to earthquake engineering, Prentice Hall, Second Edition.

Prakash, V, G H Powell y S Campbell S, (1993), "Drain-2Dx base program description and user guide", Manual, University of California.

Priestley, M, G M Calvi y M J Kowalsky, (2007), Displacement-based seismic design of structures, IUSS PRESS, Pavia, ITALY.

Qi, X y J P Moehle, (1991), “Displacement design approach for reinforced concrete structures subjected to earthquakes", Reporte UCB/EERC-91/02, University of California at Berkeley.

Reglamento de Construcciones para el Distrito Federal (2004), "Reglamento de Construcciones del Distrito Federal", Gaceta Oficial del Distrito Federal, Tomo II, No. 103-BIS, octubre.

Reyes, C (2000), "El estado límite de servicio en el diseño sísmico de edificios", Tesis Doctoral, División de Estudios de Posgrado de la Facultad de Ingeniería, UNAM.

Takahashi, N y H Shiohara, (2004), "Life cycle economic loss due to seismic damage of nonstructural elements", Proceedings of the 13th World Conference on Earthquake Engineering (CD), Paper No. 203.

Terán-Gilmore A. (1998), "Características Mecánicas y desempeño sísmico de marcos dúctiles de concreto reforzado", Memorias de XI Congreso Nacional de Ingeniería Estructural, I, 564-573.

Terán-Gilmore, A (2004), "On the use of spectra to establish damage control in regular frames during global predesign”, Earthquake Spectra, 20(3), 1-26.

Teran-Gilmore, A y J O Jirsa, (2010), "SMIS-EERI Workshop on Safe Hospital under Natural Hazards", 9th US National and 10th Canadian Conference on Earthquake Engineering (CD).

Terán-Gilmore, A, G Díaz y C Reyes, (2010), "Estudio sobre el factor de importancia utilizado durante el diseño sismorresistente de hospitales", Memorias del XVII Congreso Nacional de Ingeniería Estructural, León, Guanajuato México.

Villaverde, R (1997), "Seismic design of secondary structures: state of the art", Journal of Structural Engineering, 123 (8), 1011-1019.

Wong, B (2009), Plastic analysis and design of steel structures, Butterworth-Heinemann, Elsevier Ltd. USA 2009. 